\title{
TV-Anytime Metadata Authoring Tool for Personalized Broadcasting Services
}

\author{
Seung-Jun Yang*, Jung Won Kang*, Dong-San Jun**, Min Je Kim*, and Han-Kyu Lee* \\ *Electronics and Telecommunications Research Institute \\ **Korea Advanced Institute of Science and Technology
}

\begin{abstract}
In spite of a useful services based on TVAnytime metadata, the metadata authoring still remains as a harassing and time consuming task. In this paper, we present a design and implementation of a TV-Anytime metadata authoring tool to provide personalized broadcasting services. For easily authoring metadata, the proposed metadata authoring tool provides the following key functionalities: metadata visualization, media access, and semi-automatic method for editing segment related metadata
\end{abstract}

Index Terms - TV-Anytime, Metadata, Authoring Tool, Broadcasting Service.

\section{INTRODUCTION}

It is expected that, in the near future, multimedia data service providers will provide various kinds of data to satisfy the demand of audience in digital broadcasting environment. The TV-Anytime Forum specifies a set of metadata to be used for efficient access and browsing of broadcasting content in a personalized way [1], [2]. The metadata include plenty of information about the content that may be utilized for the electronic program guide, content-based search and browsing, and adaptation of the content to diverse terminal and network conditions. A couple of practical schemes that use the metadata in digital broadcasting can be found in [3], [4]. In spite of a useful services based on TV-Anytime metadata, the metadata authoring still remains as a harassing and time consuming task. Although some kinds of metadata are extractable automatically [5]-[7], there are still needs of human intervention for refining the results (especially, in subjective terms) and annotating manually the fields that are difficult to extract in any automated means. Therefore, an efficient authoring tool is required [8].

In this paper, we present a design and implementation of a TV-Anytime metadata authoring tool to provide personalized broadcasting services. For easily authoring metadata, the proposed metadata authoring provides the following key functionalities: metadata visualization, media access, and semi-automatic method for editing segment related metadata.

\section{Personalized Broadcasting}

The personalized broadcasting means the customized broadcast service which reflects a preference and watching pattern of a viewer, and etc. and searches a part or an entire broadcasting program and watches the broadcasting program in which a viewer wants. In case of this personalized broadcasting service, for example, the Advanced Content Guide (ACG) service, even though an user individually does not search a plurality of broadcast channels, he automatically can directly move to the desired channel through ACG and look and listen. And the function which singles out the broadcasting program such as a drama and news only the part of concern and which it can consume is possible. This personalized broadcasting service basically assumes the PDR environment having the storage. The additional information about the broadcasting program is needed so that this service can be provided [8], [9].

Metadata means 'data about data'. In broadcasting environments it means additional data to describe multimedia content, including audiovisual features for content-based retrieval as well as electronic program guide information for selecting a channel and its program. These metadata are used for searching, selecting, recording, and managing segments as well as programs in broadcasting environments. Therefore, application technologies using metadata play a very important role in providing intelligent and personalized broadcasting services in digital era [8], [9].

\section{TV-ANYTIME METADATA}

In the side of a function and system environment, the TVAnytime standard is divided into two phase of the Phase1(TVA-1) and Phase-2(TVA-2).

\section{A. TV-Anytime Phase-1}

The basic kinds of TV-Anytime metadata are content description metadata, instance description metadata, segmentation metadata and consumer metadata. Content description metadata are general information about a piece of content that does not change regardless of how the content is published or broadcast. Instance description metadata describe a particular instance of a piece of content, 
including information such as the content location, usage rules, and delivery parameters (e.g., video format) [10]-[12].

Consumer metadata, borrowed from MPEG-7, include usage history data (logging data), annotation metadata, and user preferences for personalized content service. Segmentation metadata describe a segment or groups of segments. A segment is a continuous portion of a piece of content, for example a single news topic in a news program. TVA-1 metadata is the XML document having the root element called the "TVAMain". And as shown in Fig. 1., it has the lower component of the copyrightNotice, metadataOriginationInformationTable,

classificationSchemeTable, programDescription, userDescription [10]-[12].

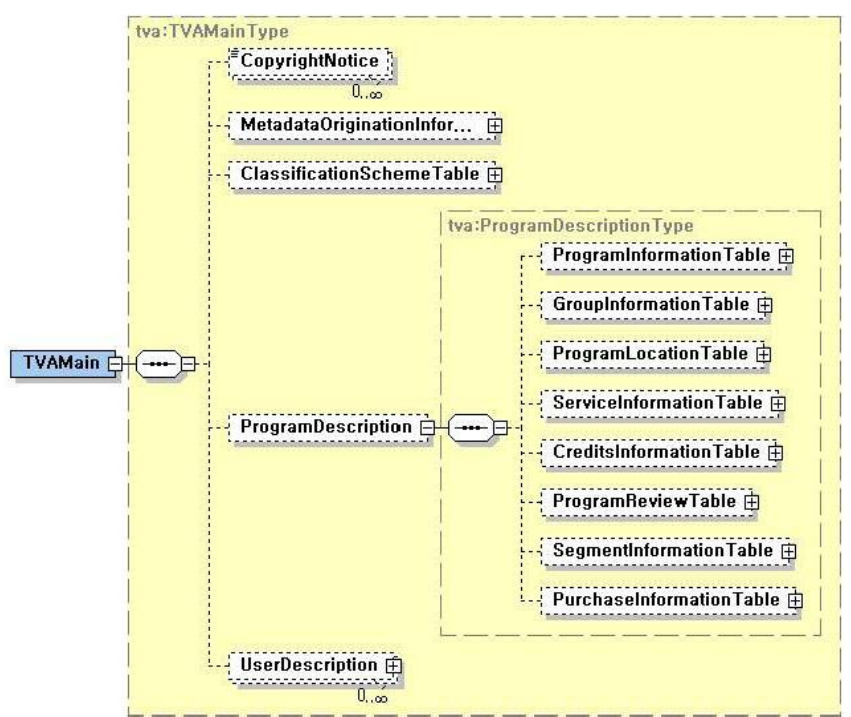

Fig. 1. Structure of TV-Anytime Phase-1 schema

\section{B. TV-Anytime Phase-2}

In addition to TVA-1, the TV-Anytime Forum has defined requirements and business models for TVA-2, which mainly deals with sharing and distribution of rich content among local storage devices and/or network digital recorders in home network environments. In the TVA-2, as shown in fig. 2., the "TVAMain" type was extended. Moreover, the standard about package, e-flyer, interstitial, sharing, remote programming, and etc were constituted [10]-[12], [13]-[16]

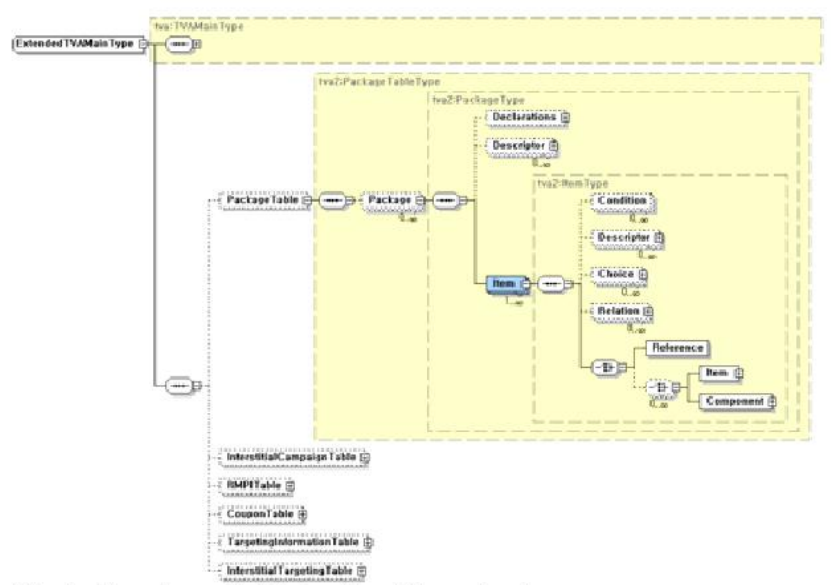

Fig. 2. Structure of TV-Anytime Phase-2 schema

\section{METADATA AUTHORING TOOL}

The proposed TV-Anytime metadata authoring tool is to provide users visual and intuitive environment for authoring content descriptive metadata. We implemented the metadata authoring tool about TVA-1 and package metadata authoring tool among TVA-2.

\section{A. TVA-1 Based Metadata authoring tool}

\section{1) Structure}

The overall structure of functional module of the TVAnytime metadata authoring tool is illustrated in Fig. 3. The implemented authoring tool provides various kinds of functionality as follows:

- Metadata loading : load existing metadata to the memory device after parsing and validation

- VCR function : support basic VCR functions including play, pause, fast-forward, and random access

- Segment extraction : be equipped with some automation tools for authoring segment metadata

- Visualization : visualize the content of metadata in an effective way and support editing in the visualized environment

- Audit : audit user interactions real-time

- Save : save results into a valid XML(eXtensible Markup Language) document

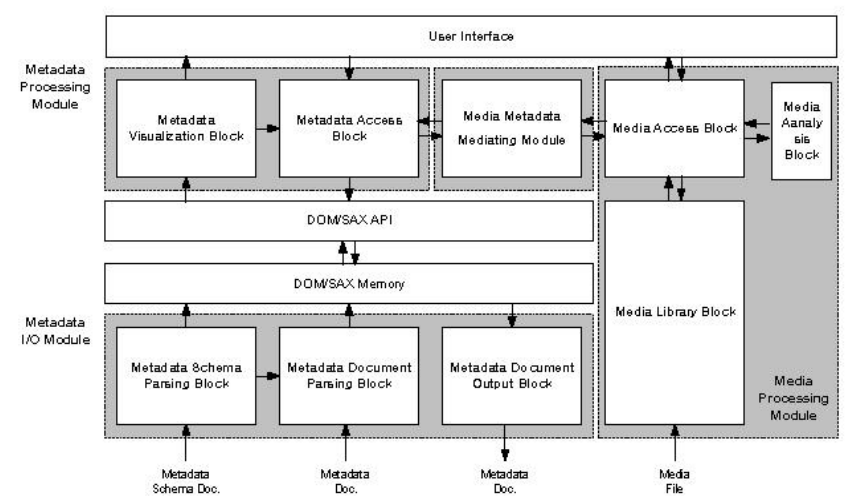

Fig. 3. Structure of the metadata authoring tool 
Fig. 3. shows a block and mutual flow of the upper level for comprising metadata authoring tool. According to a function, the authoring tool is divided into the function module as follows.

- Metadata I/O module

- Metadata process module

- Media process module

- Mediating module

The metadata $\mathrm{I} / \mathrm{O}$ module receives the metadata and schema as an input and loads the memory. It is the function module which converts edited metadata into XML and which it outputs. This module is comprised of schema parsing block, and the metadata parsing block and metadata output block.

The metadata process module visualizes the metadata loaded in the DOM through the user interface. And the DOM is changed according to the user interface, or the editing message inputted from the mediating module between AV media and metadata. The metadata process module is comprised of the metadata visualization block and metadata access block.

The media process module receive the MPEG-1 media file as an input and access this and playback. And it has an interface with the mediating module in order to operate metadata relating to the particular section of a media. The AV media process module is comprised of the media access block, and the media analysis block and media library block. In the developed authoring tool, the algorithm automatically detecting the boundary of the content is supported.

The mediating module mediates between the metadata access block and media access block. The playback message for reproducing a media is delivered to the media access block with metadata relating to the specified section of a media. And if the event for editing metadata relating to the specified section of a media through the media access block is generated, the corresponding editing message is delivered to the metadata access block.

\section{2) Interface}

The implemented metadata authoring tool provides the metadata visualization, editing, media access, and semiautomatic method for editing segment related metadata. The implemented authoring tool is seen in fig. 4 .

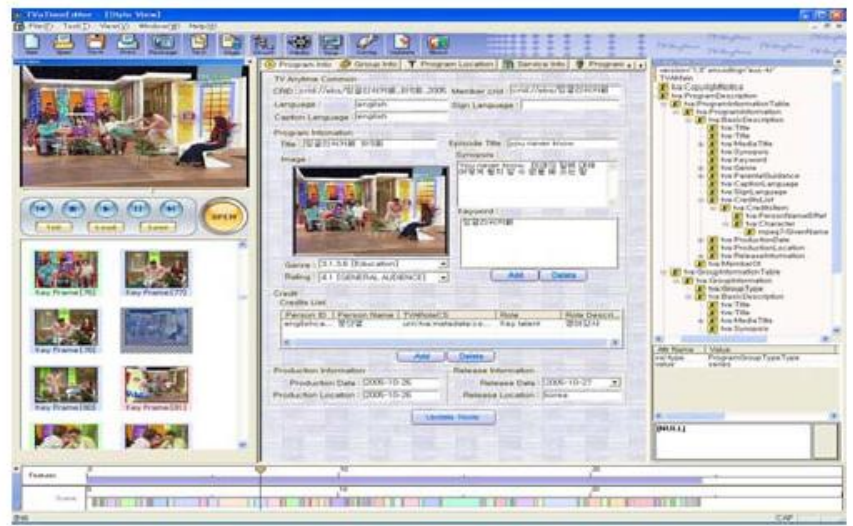

Fig. 4. Screen shot of Interface

The developed metadata authoring tool provides three types of visualization. The first one is a pure text, the most naïve form of visualization, where the XML tags are treated the same as the contents. The second is a tree style visualization for the hierarchical structure of the metadata and a spreadsheet style presentation for the contents of a selected element. Such visualization method is useful to search the particular element or browse the whole metadata structure and to insert the new node, which it is unable to express in the template based visualization. The last one is a template-based visualization, providing the most intuitive editing condition. This method provides the environment editing metadata easily authored to the user without the special knowledge about XML. According to an application, if the frequently used metadata element is prescribed, since data type about element or the attribute values is shown in the template defined in advance a user adds the content that it just wants or it can modify. The authoring tool automatically converts this into the XML document when the storage of the document in which a correction of a user is completed is desired.

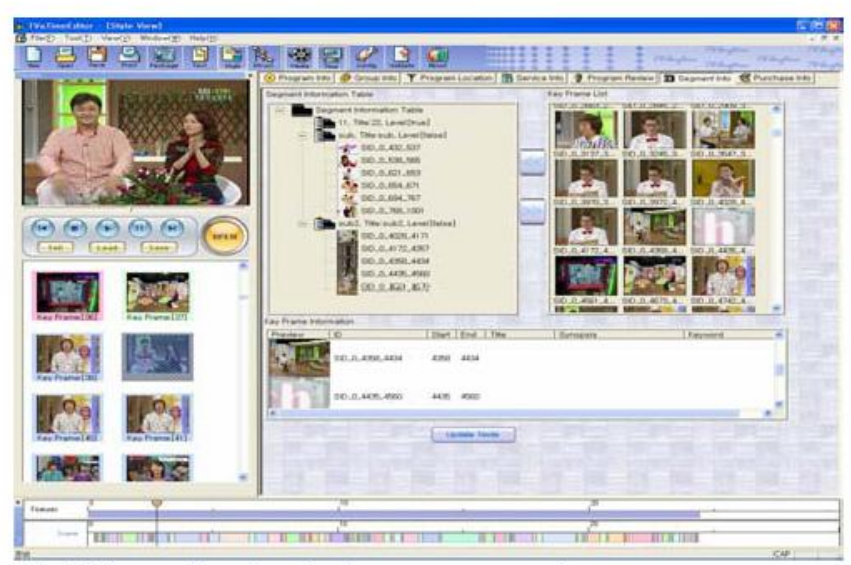

Fig. 5. Screen shot of authoring segment metadata

For making it easy to edit media specific metadata, various kinds of accessing methods are provided: basic VCR functions (e.g., play, pause, stop, fast-forward, etc.), 
time line, or key frames (visual index). Fig. 5. shows the user interface of the developed metadata authoring, where the part of media specific extension is also included.

Visual index generation is closely related to the shot boundary detection [5] and key frame extraction [6], which have been active research issues for the past few years. We can make use of these approaches. Let us briefly present the shot boundary detection method used for the generation of segment metadata in the proposed framework. We use color and edge features for detecting shot boundaries and perform all of the operations in the compressed domain [7].
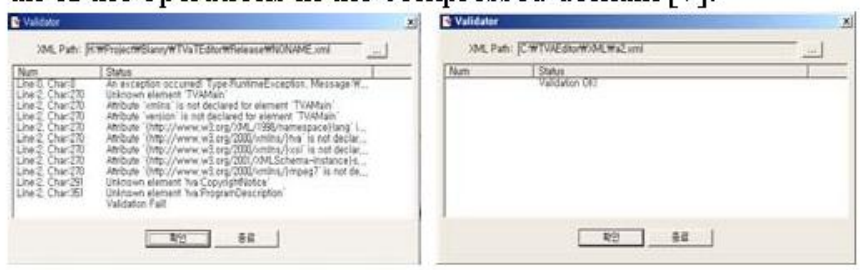

Fig. 6. Validation tool of metadata

Fig. 6. shows the metadata verification tool. The metadata validation tool inspects whether metadata were validated in the TVA schema or not.

\section{B. Package Metadata authoring tool}

With the package metadata authoring tool, a user easily generates the package metadata following the schema of TVA-2.

\section{1) Interface}

With the package authoring tool, each node of a schema can be easily added by using the interface of a tree-view. And it is implemented in order to look into the detailed information of each node easily.

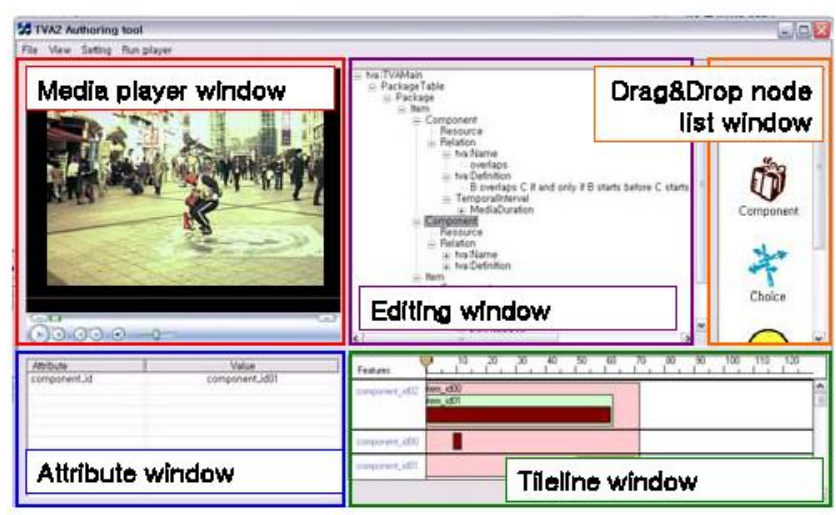

Fig. 7. Interface of package metadata authoring tool

The some kind item frequently used for the metadata generation including an item, a component, a choice, and etc can be easily added using the drag \& drop. In addition, the temporal relation between a component and an item can be easily displayed as a timeline and it can be easily modified.

Moreover, a text view is supported besides the XML code view, and a tree view to be easy to look at a code and modify and an editing is possible in not only the editing at the environment which is user friendly but also the XML source code level. The generated package metadata operated with the package player and easily verified an operation.

If it examines, the editorial window of the respectively different three type of the $\mathrm{XML} /$ code/text is provided the XML output. And the environment which produces metadata through the respectively different interface and which it edits according to the selection of a user is provided.

The attribute window shows the attribute value of the XML node selected in the XML tree. In the upper end, the already added attribute value is indicated and attributes can add are expressed in a bottom and a user inputs only the real value.

The media player can reproduce the resource of an image and AV content in order to confirm the resource of the package metadata. A timeline indicates components included in a package with the time information.

Moreover, the timeline of each component provides a function of directly changing a relation through the drag operation.

In the Drag \& Drop node list on the window, item, component, choice, and condition that are frequently used to author a package are iconized and, with this feature, a user can easily add a new node in the Drag \& Drop node list.

Whenever a user selects, drags, and drops an icon that the user wants to newly add in order to author a package, a user easily can produce a new node.

\section{2) Package player}

The package metadata player is used in order to express the interpretation about the playback of a media and meta data, and an application for the verification of the package metadata generated with the proposed package metadata authoring tool visually.

The package metadata player interprets the temporal/spatial relationship within metadata and reproduces the contents according to the correlation of a component and item. It consumes the contents according to the input from a user by showing the selection elements to a user through user interface.

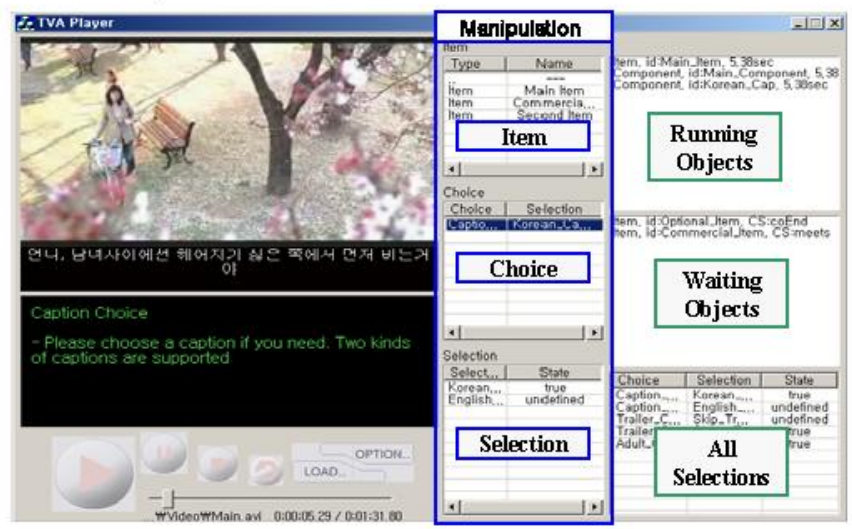

Fig. 8. Package player 
Fig. 8. shows the package metadata player. The video window reproduces a video content by interpreting a package metadata. The subtitle window outputs the closedcaption related to a video.

The description window outputs the description including an item or a choice, and etc. that are selected. The manipulation window manages the load of the metadata file, the playback of a video, and etc.

In the item window, a package or the selectable item is shown up. If packages or an item within a list is selected, that can be consumed.

In the choice window, the selectable choice and the selection selected by a choice are indicated together. If the choice within a list is selected, it shows up in the selection window in the selection list of the selected choice.

In the selection window, the selection within the choice selected in the choice window is indicated. The state of a selection on a list can be changed and the interpretation of metadata gets accomplished according to the state when it is changed.

In the progressing list window, the information registered in running object list is described.

In the atmospheric list window, the information registered in waiting object list is specified.

In the selection list window, not only selected choice but also selected all selections are indicated and can describe the current state.

\section{EXPERIMENT AND RESULT}

The implemented TV-Anytime metadata authoring tool consists of a media player, template-based editor, tree-based view/editor, time line, and so on. And it provides various kinds of metadata creation and validation function.

By using the developed metadata authoring tool, we authored broadcasting content metadata. And the service using this metadata was confirmed through the together developed customized broadcasting terminal platform(STB).

Fig. 9.-12. shows the received result screen about the advanced EPG service, my Program service, advanced PVR service, bi-directional metadata service which can be offered through an experiment.

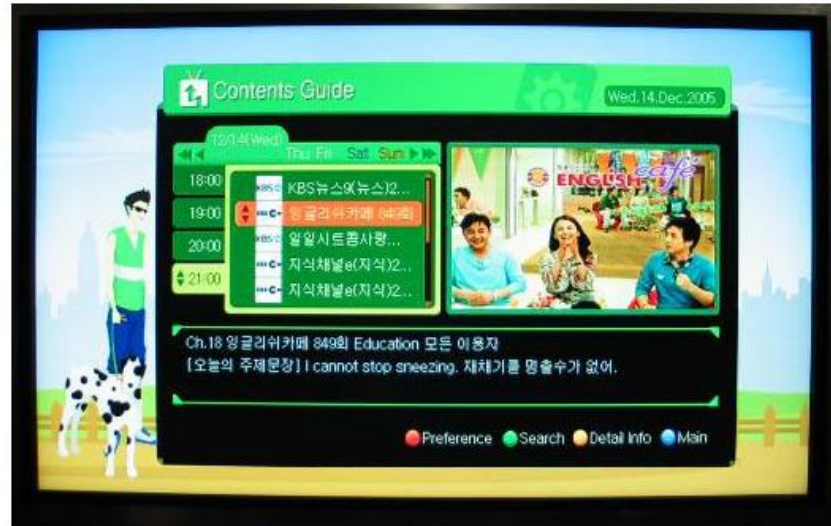

Fig. 9. Advanced EPG service

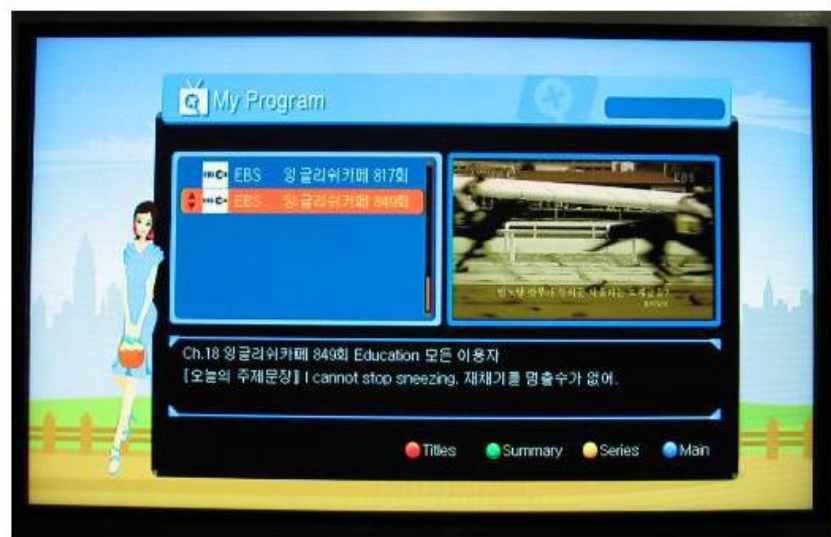

Fig. 10. My Program service

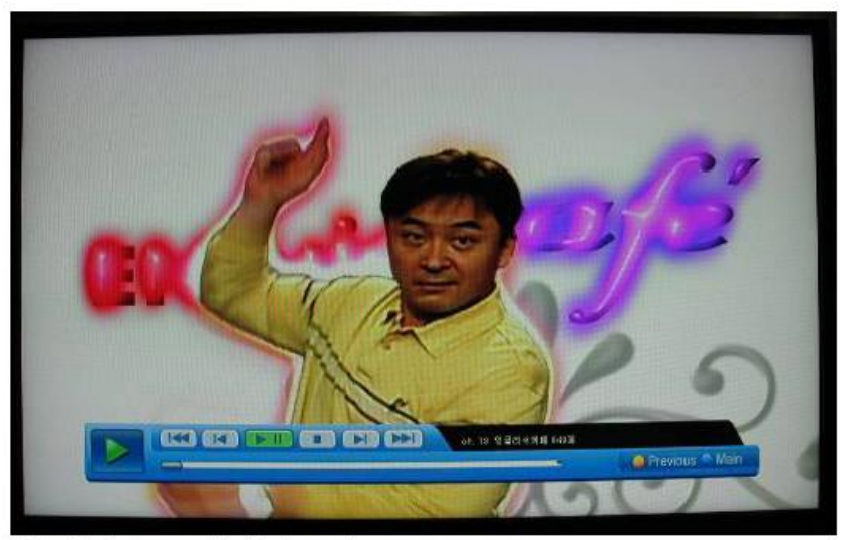

Fig. 11. Advanced PVR service

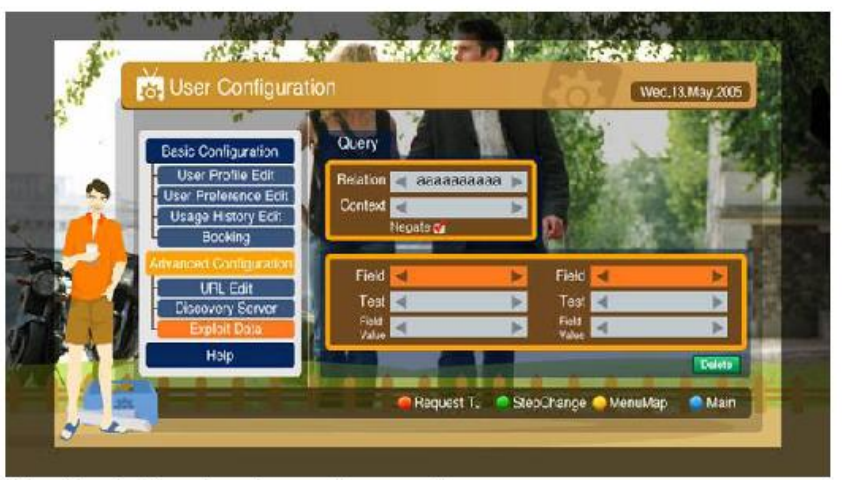

Fig. 12. Bi-dir ectional metadata service 


\section{CONCLUSION}

In this paper, we present a visual tool for authoring TVAnytime metadata. The proposed visual metadata authoring tool provides the convenience of user at authoring step. We expect that this authoring tool can be applied to various personalized broadcasting services such as DTV, DCATV, DMB and so on.

\section{ACKNOWLEDGMENT}

This work was supported by the IT R\&D program of MIC/ITTA.[2006-S082-01, Development of digital multimedia broadcasting technology for personalized broadcasting]

\section{REFERENCES}

[1] The TV-Anytime, "TV-Anytime Forum," http://wrww.tv-anytime.org/, 2004

[2] The TV-Anytime Forum, Requirement Series: RQ001v2.0 on Phase 2 Business Model: TV 140

[3] A McParland et al., "myTV: A practical implementation of TV Anytime on DVB and the Internet," in Proc. IBC2001, pp. 116-125, Amsterdam, the Netherlands, Sept. 2001.

[4] K. Kang et al., "Metadata broadcasting for personalized service: a practical solution," ETRI Joumal, vol. 26, no.5, pp. 452-466, Oct, 2004

[5] A Hanjalic, "Shot-boundary detection: unraveled or resolved?," IEEE Trans. CSVT, vol. 12, pp. 90-105, Feb. 2002.

[6] H. S. Chang, S. Sull, and S. U. Lee, "Efficient video indexing scheme for content-based retrieval," IEEE Trans. CSVT, vol. 9, pp. 1269 1279 , Dec. 1999

[7] H. S. Chang and K. Kang, "A fast scheme for classifying block edge patterns in DCT-compressed images," ETRI Technical Memorandum, Allg. 2002.

[8] H. S. Chang, S. J. Yang, J. G. Kim and K. Kang, "Authoring content descriptive metadata in a visual framework," ITC-CSCC'2003, vol. 2, pp. 1254-1257. July. 2003

[9] Kyeongok Kang Jae-Gon Kim, Heekyung Lee, Hyun Sung Chang Seung-Jun Yang, Young-tae Kim, Han-kyu Lee, and Jinwo ong Kim, "Metadata Broadcasting for Personalized Service: a Practical Solution", ETRI Joumal, vol.26, no. 5 , pp.452-466. 2004.

[10] ETSI TS $102822-1$ V1.3.1: Broadcast and On-line Services: Search, select, and rightful use of content on personal storage systems ("TV. Anytime"); Part 1: Benchmark Features, January, 2006.

[11] ETSI TS 102 822-2 V1.3.1: Broadcast and On-line Services: Search, select, and rightful use of content on personal storage systems("TV Anytime"); Part 2: System description, January, 2006.

[12] ETSI TS 102 822-3-1 V1.3.1: Broadcast and On-line Services: Search, select, and rightful use of content on personal storage systems("TV Anytime"); Part 3: Metadata; Sub-Part 1: Phase 1-Metadata schemas, January, 2006

[13] ETSI TS 102 822-3-3V1.1.1: Broadcast and On-line Services: Search, select, and rightful use of content on personal storage systems("TV. Anytime"); Part 3: Metadata; Sub-Part 3: Phase 2 - Extended Metadata Schema, January, 2006

[14] Heekyung Lee, Jae-Gon Kim, Jin Soo Choi, and Jinwoong Kim, "Package Schema for Targeting \& Synchronization," TV-Anytime Form, AN602, March 2004. [Online]. Available: $\mathrm{ftp} /$ /tva:tva@ftp.bbc.co.uk/pub/Contributions/

[15] The TV-Anytime Forum, Requirement Series: RQ001v2.0 on Phase 2 Business Model: TV 140

[16] TV-Anytime Forum, Working Document: Packaging and Targeting (2004, June). [Online]. Available: ftp//tva:tva@ttp.bbc.co.uk/pub/Plenary/WD997.zip.

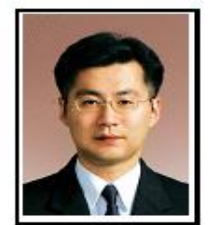

Sang-Jun Yang received the BS degree in computer science from Suncheon National University, Korea in 1999 and the MS degree in computer science from Chonnam National University, Korea in 2001. Since 2001 , he has been a senior member of research staff in Broadcasting Media Group of ETRI, where he has developed an advanced digital television technology such as data broadcasting and personalized broadcasting. He has involved in making domestic personalized broadcasting standard, transmission and reception standard for terrestrial personalized broadcasting, as a member in Telecommunications Technology Association. His research interests include TV-Anytime metadata, personalized broadcast systems, and metadata generation.

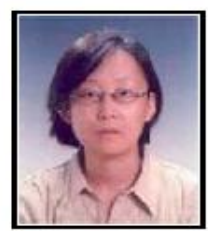

Jung Won Kang received the BS and MS degree in Electrical Engineering in 1993 and 1995, respectively, from the Hankuk Aviation University, Seoul, South Korea. She received the Ph. D degree in Electrical and Computer Engineering in 2003 from Georgia Institute of Technology, Atlanta, GA. Since 2003, she has been a Senior Member of Research Staff in the Broadcasting Media Research Group of Electronics and Telecommunications Research Institute (ETRI), Korea. Her research interests are in the area of video signal processing, video coding, and video adaptation.

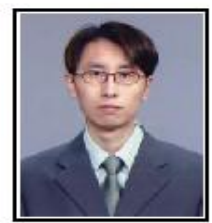

Dong-San Jun received his BS degree in electrical engineering from Pusan National University, Pusan, Korea in 2002, and his MS degree in electrical engineering from Korea Advanced Institute of Science and Technology (KAIST), Daejeon, Korea, in 2004. He was a researcher with the Electronics and Telecommunications Research Institute (ETRI) in Korea from 2004 to 2007. He is currently studying for his $\mathrm{PhD}$ degree in electrical engineering with the Korea Advanced Institute of Science and Technology (KAIST). His Current research interests include image computing system, video compression, pattern recognition, and TVAnytime metadata

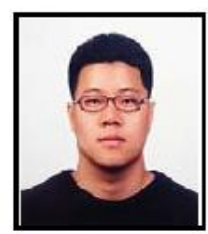

Minje Kim is a member of research staff working on multimedia technologies for interactive and intelligent digital broadcasting in ETRI, Korea He got his B.S degree from Ajou University and M.S. degree from Postech, in computer science, in 2004 and 2006 , respectively. His research is based on statistical machine learning and he is widening his application area from signal processing, multimedia broadcasting and user-friendly multimedia consuming systems.

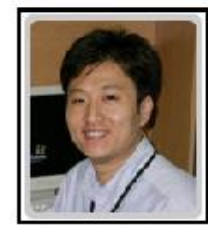

Han-kyu Lee received the BS and MS degrees in electronics engineering from Kyungpook National University, Daegu, Korea, in 1994 and 1996 repectively. In 1996, he joined Electronics and Telecommunications Research Institute(ETRI) Daejeon, Korea, where he has worked for research and development of broadcasting and multimedia technologies. Since 2005, he has served as team leader for Personalized Broadcasting Research Team of ETRI. His main research intrestes are in the areas of signal processing, intelligent and interactive systems for multimedia. 